\section{Sharing the benefits in IBD}

\section{By Michael J. Haas, Senior Writer}

For at least two decades it has been hypothesized that deficiencies in commensal bacteria-the beneficial gut flora-contribute to or potentially cause autoimmune disorders such as inflammatory bowel disease. But the molecular mechanisms by which these bacteria confer their benefits, and how deficiencies might contribute to disease, are poorly understood.

In a paper in Nature, researchers at Harvard Medical School and the California Institute of Technology have elucidated the first such molecular mechanism. ${ }^{1}$ The group found that polysaccharide A (PSA), a molecule produced by the commensal bacterium Bacteroides fragilis, prevented inflammatory bowel disease (IBD) in mice by inducing an anti-inflammatory effect in the gut. The team was led by Dennis Kasper, professor of medicine, microbiology and molecular genetics at Harvard.

Company and institution representatives contacted by SciBX agreed that the team's findings advance the understanding of how commensal bacteria work. But they also said it was unclear whether the findings would apply to IBD in humans, both because of the animal models used and because a link between $B$. fragilis and IBD has yet to be established in humans.

Additional hurdles to commercialization include establishing which IBD patients-if any - would benefit from treatment with PSA or B. fragilis and determining how to deliver either the molecule or the bacterium to the appropriate section of the GI tract.

\section{Preventing colitis}

PSA, one of several polysaccharides secreted by B. fragilis, was first identified in 1992 by Kasper and other colleagues at the National Research Council of Canada. ${ }^{2}$ A 2005 study by a team at Harvard Medical School, also led by Kasper, showed that PSA modulated T helper type 1 and type 2 cell functions in the GI tract of animals. ${ }^{3}$

Building on that earlier work, the latest work by Kasper and his team explored the mechanism of PSA in three different mouse models.

First, the team transferred antigen-naïve $\mathrm{T}$ cells into mice bred to lack commensal bacteria. The mice developed colitis when colonized with the opportunistic bacterium Helicobacter hepaticus. However, colitis did not develop when the mice were colonized with both $H$. hepaticus and B. fragilis.

Similar results were obtained when only PSA was given to the $H$. hepaticus-colonized mice. Histological analyses of the colons of PSAtreated mice found no signs of inflammatory cells.

Second, the team showed that PSA prevented the onset of inflammation in a mouse model of chemically induced colitis. This model mimics the initial, acute inflammation that, if unresolved, can lead to the chronic inflammation that characterizes IBD in humans.

Third, the team showed that neither $B$. fragilis nor PSA prevented $H$. hepaticus-induced inflammation in IL-10 knockout mice.

Lastly, experiments on murine cell lines showed that PSA stimulated $\mathrm{CD}^{+} \mathrm{T}$ cells that produce IL-10, an anti-inflammatory agent that suppresses two proinflammatory molecules involved in IBD: IL-17 and IL-23.

"These results constitute the first reported evidence of a symbiotic bacterial molecule that networks with the immune system to coordinate anti-inflammatory responses," according to the authors. They suggest the findings could lead to new therapies for IBD based on PSA or other immunomodulating bacterial molecules.

"There are clearly examples of probiotics and many accounts of their health benefits," Sarkis Mazmanian, coauthor and spokesperson for the research team, told SciBX. "However, in all cases, the mechanisms are not known and the precise immune responses are not well documented."

The Nature paper begins to fill that gap, he said.

Mazmanian is an assistant professor of biology at Caltech.

Cynthia Sears, professor of medicine at Johns Hopkins University, agreed that the Nature paper added clarity to the "exceptionally confusing literature" on probiotics. "Identifying a molecule from a benign organism is a step forward" in understanding the precise actions of commensal bacteria, she said.

Marie Kosco-Vilbois, CSO of NovImmune S.A., said "the work is very exciting, as the researchers have been able to focus on the role of a particular polysaccharide that provides key benefits" in preventing colitis.

Catherine Walker, program leader for anti-inflammatory and cardiovascular therapeutics at Novogen Ltd., agreed with Sears and Kosco-Vilbois. She said the paper "helps explain the promising findings for the use of probiotics as therapeutics agents."

Walker noted the paper complements a recent study that showed an anti-inflammatory benefit of delivering IL-10-expressing genes, via nanoparticles, in an animal model of IBD. ${ }^{4}$

\section{Of mice and men}

A significant question is whether the models used in the Nature study are relevant to IBD in humans. 
Dominick Auci, director of allergy, autoimmunity and inflammation at Hollis-Eden Pharmaceuticals Inc., said it was unclear how the team's findings in mice would play out in IBD patients.

"The IBD model they use is really artificial," he said, noting that repopulating germ-free mice with bacteria, then transferring naïve $\mathrm{T}$ cells into those immune-compromised animals, does not represent what happens in "the real world."

"Also," Auci said, "this is more a model of acute inflammation, whereas IBD is a chronic condition."

Hollis-Eden's Triolex NF- $\kappa \mathrm{B}$ inhibitor is in Phase II trials to treat IBD and type 2 diabetes, and it is in preclinical development for cystic fibrosis (CF). The company plans to start a Phase II trial of Triolex to treat rheumatoid arthritis (RA) this year.

Sears also thinks the mouse models used in the paper were "pretty artificial. There are over 35 murine models of IBD, most of which are mice," deficient in a particular protein, such as IL-10, she said.

Like Auci, she noted that Kasper's team used models that represented acute disease states.

"Whether slower onset, more chronic colitis will respond similarly to these rather artificial models remains to be seen," Sears said, because the mechanisms of acute and chronic inflammation may be different and the chronic state may be harder to treat.

Mazmanian disagreed. "The transfer model, in my opinion, is one of the most representative animal models for human IBD currently available," he said. "In addition, it is well accepted and widely used."

The main problem with animal models of IBD, he said, is "how little we actually know about many of the characteristics of the human disease."

Kosco-Vilbois did note that "the experi-

\section{"If you could subtype the disease and link it to a particular bacterial strain or bacterial imbalance, you might be able to choose better, more directed therapies."}

- Cynthia Sears, Johns Hopkins University
Indeed, there are multiple challenges in linking a deficiency in any one type of bacteria to a significant fraction of IBD patients. First, the human gut is home to hundreds of species of bacteria-not all of which have been unequivocally identified. Second, the small and large intestines are home to different populations of bacteria.

Moreover, IBD encompasses two distinct conditions-Crohn's disease and ulcerative colitis (UC) - that involve the small intestine and large intestine, respectively.

"If you could subtype the disease and link it to a particular bacterial strain or bacterial imbalance, you might be able to choose better, more directed therapies" to treat an individual patient, Sears said.

Levinus Dieleman, associate professor of medicine at the University of Alberta, thinks the link between IBD and B. fragilis deficiency is tenuous. He said postulating such a link assumes that many, if not all, IBD patients share a deficiency in one of the more common types of commensal bacteria. "But populations of gut flora vary from one person to the next, so it's very hard to make any general assumptions bout them," he said.

The situation is similar to that of probiotics, Dieleman added. "Some people respond to probiotics more than others, but we don't know whether this is because they are deficient in that particular organism."

Dieleman expects that IBD patients who are not deficient in $B$. fragilis would not see as much benefit from $B$. fragilis or PSA therapy as patients who were deficient. He said any clinical trials of these therapies would first need to identify IBD patients deficient in the bacteria to predict who would benefit.

Sears agreed that the question was important: "If you have a patient with IBD, and they carry B. fragilis, will flooding [the] gut with PSA still work to inhibit inflammation?" mental findings reinforce the nature of colitis in animal models of the human disease" by highlighting the interplay of T cell dependence, IL-10's anti-inflammatory action and the role of bacteria in disease susceptibility. "It is impressive that they can use the three different models to establish proof of concept," she said.

Still, Kosco-Vilbois said she would have liked to see experiments in human cell lines to confirm the findings. Also, she said, "as the exposure to PSA is short, one wonders what immunogenicity issues would arise with repeated treatment."

NovImmmune has NI-0401, an anti-CD3 mAb, in Phase I/II trials to treat Crohn's disease. The company expects to start a Phase II/III trial in type 1 diabetes in 4Q08.

\section{Fragile connections}

Even if the mouse models are representative of the human disease state, an open question is whether deficiency in a single type of gut bacteria, such as $B$. fragilis, or a bacterial product, such as PSA, contributes to IBD in humans.

A 2007 study by a University of Colorado team explored the link between gut bacteria deficiencies and IBD. ${ }^{5}$ The researchers found that $25-30 \%$ of IBD patients were deficient in species of the Bacteroides genus, but did not specifically identify whether $B$. fragilis was one of them, noted Sears.
Sears would have liked to see Kasper's team conduct experiments in normal mice to address this. She said such mice would probably display some PSA in their guts already, so it would be interesting to determine whether giving them additional PSA would have an increased anti-inflammatory effect.

Novogen's Walker agreed that the relationship of B. fragilis and PSA to the induction of IL-10 in humans needs to be explored. Novogen recently completed Phase I trials of NV-52, a selective thromboxane

Hollis-Eden's Auci doubted whether any single probiotic or bacterial product would be a truly beneficial therapy in a disease like IBD, noting that even in healthy individuals, gut flora populations are not stable. "You ingest lots of bacteria every day and your gut flora changes dramatically," he said.

Thus, Auci does not think that autoimmune disorders like IBD or allergies are driven by a simple deficiency of a particular commensal organism. "Simply supplying a particular bacterium or bacterial product is unlikely to be curative or even a good general therapeutic strategy," he said.

He added: "Even if, in certain patients, a particular disease is caused by the lack or expansion of a particular organism, good luck in trying to figure out which one it is and how it relates to the disease state" of a given patient. synthase inhibitor, to maintain remission of IBD. 


\section{TARGETS \& MECHANISMS}

\section{Effective delivery}

Even if a connection between IBD and a B. fragilis deficiency is found, there are still hurdles to delivering either the bacterium or PSA as a therapeutic agent.

Sears said that delivering the bacteria as a probiotic would get it past the stomach and small intestine to the colon, "but you wouldn't expect this to have an effect on Crohn's disease, which affects the small intestine. B. fragilis itself only colonizes the colon, not the small intestine."

Unlike the bacteria, pure PSA might have an effect in Crohn's disease, she added. But for UC "the translational question is whether you can feed PSA to someone and expect it to get all the way to the colon and stick."

Dieleman noted that PSA would have to be carefully dosed because of its anti-inflammatory properties. "You would not want to administer this when the patient has a GI infection-C. [Clostridium] difficile, for instance-because you might suppress the necessary inflammatory response" to the infection, he said.

Overall, Auci said, attempts to harness the interactions between commensal bacteria and the host immune system, as highlighted in the Nature paper, are "intensely interesting with a huge potential, especially in IBD and allergic disease. But it is difficult in practice and not exactly low-hanging fruit."

Mazmanian agreed with Auci that the findings are a long way from yielding a preventive or therapeutic agent for IBD. For now the team is focused on elucidating the precise mechanism by which PSA induces IL-10-producing T cells.

"How the structure of PSA links to the mechanism is a mystery," he told SciBX. "PSA is taken up by specific intestinal dendritic cells [DCs] and internalized. We think this initiates the immune response, but we don't know which type of DC or which receptors are involved.
The DC type is relevant, because that would indicate what kind of immune response is induced."

Mazmanian declined to speculate on how PSA or B. fragilis might be administered therapeutically. "This is too far down the road to extrapolate from our current work," he said. "First we want to understand the basic mechanism."

Until then, he said, the team does not think the findings are ready for commercial development. "But a biotech might look at this and decide to give it a try."

Harvard Medical School and Caltech have applied for a patent on the findings.

\section{REFERENCES}

1. Mazmanian, S., et al. Nature; published online May 29, 2008; doi:10.1038/nature07008

Contact: Dennis Kasper, Harvard Medical School, Boston, Mass. e-mail: dennis_kasper@hms.harvard.edu

Contact: Sarkis Mazmanian, California Institute of Technology, Pasadena, Calif. e-mail: sarkis@caltech.edu

2. Baumann, H. et al. Biochemistry 31, 4081-4089 (1992)

3. Mazmanian, S. et al. Cell 122, 2-4 (2005)

4. Bhavsar, M.D. \& Amiji, M.M. Gene Ther.; published online April 17, 2008; doi:10.1038/gt.2008.67

5. Frank, D. et al. Proc. Natl. Acad. Sci. USA 104, 13780-13785 (2007)

\section{COMPANIES AND INSTITUTIONS MENTIONED}

California Institute of Technology, Pasadena, Calif. Harvard Medical School, Boston, Mass. Hollis-Eden Pharmaceuticals Inc. (NASDAQ:HEPH), San Diego, Calif. Johns Hopkins University, Baltimore, Md. National Research Council of Canada, Ottawa, Ontario, Canada Novlmmune S.A., Geneva, Switzerland Novogen Ltd. (ASX:NRT; NASDAQ:NVGN), North Ryde, Australia University of Alberta, Edmonton, Alberta, Canada University of Colorado, Boulder, Colo. 